\title{
Morphometry of the corpus callosum in patients with questionable and mild dementia
}

\author{
A Hensel, H Wolf, F Kruggel, S G Riedel-Heller, C Nikolaus, T Arendt, H J Gertz
}

J Neurol Neurosurg Psychiatry 2002;73:59-61

Previous studies have shown a reduction in corpus callosum area in advanced Alzheimer's disease, but it is unclear whether callosal atrophy is present in the transitional phase between health and the onset of dementia. The aim of this study was to investigate whether callosal atrophy is present in subjects with questionable and mild dementia and to assess the diagnostic value of callosal measures. In 83 subjects aged 72 to 85 years (33 normal controls, 27 patients with questionable dementia, 23 with mild Alzheimer's disease), magnetic resonance images were recorded and the mid-sagittal callosal area measured. Significant differences were found between normal controls and mild dementia. In subjects with questionable dementia callosal size was intermediate between normal controls and mild Alzheimer's disease. However, callosal measures were unsuitable for diagnostic differentiation between healthy subjects, subjects with questionable dementia, and subjects with mild Alzheimer's disease. The severity of white matter changes did not differ between the groups.

$\mathrm{T}$ he corpus callosum, the main interhemispheric fibre connection, has been shown to atrophy in patients with Alzheimer's disease. ${ }^{1}$ Most studies have involved rather advanced cases, with moderate to severe dementia. Little is known about how early callosal atrophy occurs in Alzheimer's disease. Using magnetic resonance imaging, we measured the mid-sagittal corpus callosum area in patients with questionable and mild dementia and assessed the diagnostic value of callosal measurements in the transition from healthy aging to mild Alzheimer's disease.

\section{METHODS}

The sample included 83 right handed subjects within an age range of 72 to 85 years. Written consent was obtained from all participants.

Three groups were formed according to clinical dementia rating $(\mathrm{CDR}),{ }^{2}$ which comprises scales for memory, orientation, judgement, and problem solving, community affairs, home and hobbies, and personal care. The resulting groups comprised 33 healthy controls $(C D R=0), 27$ subjects with questionable dementia $(\mathrm{CDR}=0.5)$, and 23 subjects with mild dementia $(\mathrm{CDR}=1)$.

Subjects with questionable dementia typically suffered from mild forgetfulness, were fully oriented, had no or slight impairment in social functions, and did not meet ICD-10 dementia criteria.

All subjects with mild dementia had late onset of the disease. According to ICD-10 research criteria, 19 were diagnosed as having Alzheimer's disease, and the remaining four as having mixed Alzheimer's disease (two had a history of stroke with temporary paralysis, two had a stroke-like event).
None of the subjects with mixed Alzheimer's disease fulfilled the NINDS-AIREN criteria for vascular dementia. ${ }^{3}$

None of the subjects had a history of major psychiatric illness, Parkinson's disease, or substance abuse. Subjects were recruited from the Leipzig longitudinal study of the aged, $(\text { LEILA } 75+)^{4}$ and from the local memory clinic. All subjects were investigated clinically. Cognitive skills were assessed on the basis of the test performance part of the Structured interview for the diagnosis of Alzheimer's dementia and multi-infarct dementia (SIDAM), which includes all 30 items of the mini-mental state examination (MMSE) ${ }^{6}$ In cases with cognitive deficits, a collateral source was interviewed.

Subjects were investigated with a volumetric Tl weighted MPRAGE sequence on a 1.5 Tesla tomograph (Siemens Vision, time of repetition (TR) $11.4 \mathrm{~ms}$, time of echo (TE) $4.4 \mathrm{~ms}, 128$ slices, matrix $256 \times 256$, voxel size $0.9 \times 0.9 \times 1.5 \mathrm{~mm}$ ) and a T2 weighted sequence (TR 5016; TE 132; 19 slices: thickness 5 $\mathrm{mm}$; orientation transverse; $1 \mathrm{~mm}$ gap; matrix $357 \times 512$ ).

Datasets were analysed using the BRIAN system. ${ }^{7}$ The volumetric datasets were aligned with the stereotactical coordinate system, ${ }^{8}$ using anterior and posterior commissure as reference points and scaled to an isotropical voxel resolution of 1 $\mathrm{mm}$. We selected the sagittal slice best representing the midsagittal plane (little or absent grey matter and a visible septum pellucidum). The corpus callosum was divided into five subregions as suggested by Weis et al (fig 1). ${ }^{9}$ Callosal subregions were outlined manually (by $\mathrm{CN}$ ). Pixels of high signal intensity were assigned to the corpus callosum. Signal intensity was assessed visually without using an objective threshold. To evaluate measurement reproducibility, 15 images were independently outlined by a second rater $(\mathrm{AH})$. The mean (SD) proportional difference between the two measures was $5.5(3.5) \%$, the correlation coefficient was 0.918 $(\mathrm{p}<0.001$, one tailed $)$. Additionally, the mid-sagittal intracranial area was outlined manually following Pantel's technique. ${ }^{1}$ White matter changes were assessed on the basis of $80 \mathrm{~T} 2$ weighted sequences. Three $\mathrm{T} 2$ recordings failed.

All statistical computations were performed using the SPSS package for Windows (version 8.0.0). The significance level was set at 0.05 for all analyses. We used adjusted callosal measures (raw measures divided by mid-sagittal intracranial area, multiplied by 100) in all analyses. Repeated measures analyses of variance (ANOVA) with LSD post hoc analyses were performed to assess group differences, sex differences, and interaction effects. Age was entered as a covariate. The diagnostic value of the total adjusted callosal area was investigated by discriminant analysis.

\section{RESULTS}

ANOVA testing with group and sex as factors showed that there was no significant difference in age $(\mathrm{F}[2,77]=0.779$, $\mathrm{p}=0.463)$ or years of education $(\mathrm{F}[2,77]=2.457, \mathrm{p}=0.092)$ between the groups, but a significant difference in MMSE scores $([\mathrm{F} 2,77]=159, \mathrm{p}<0.001)$.

All five callosal segments were smallest in patients with mild dementia, followed by patients with questionable 


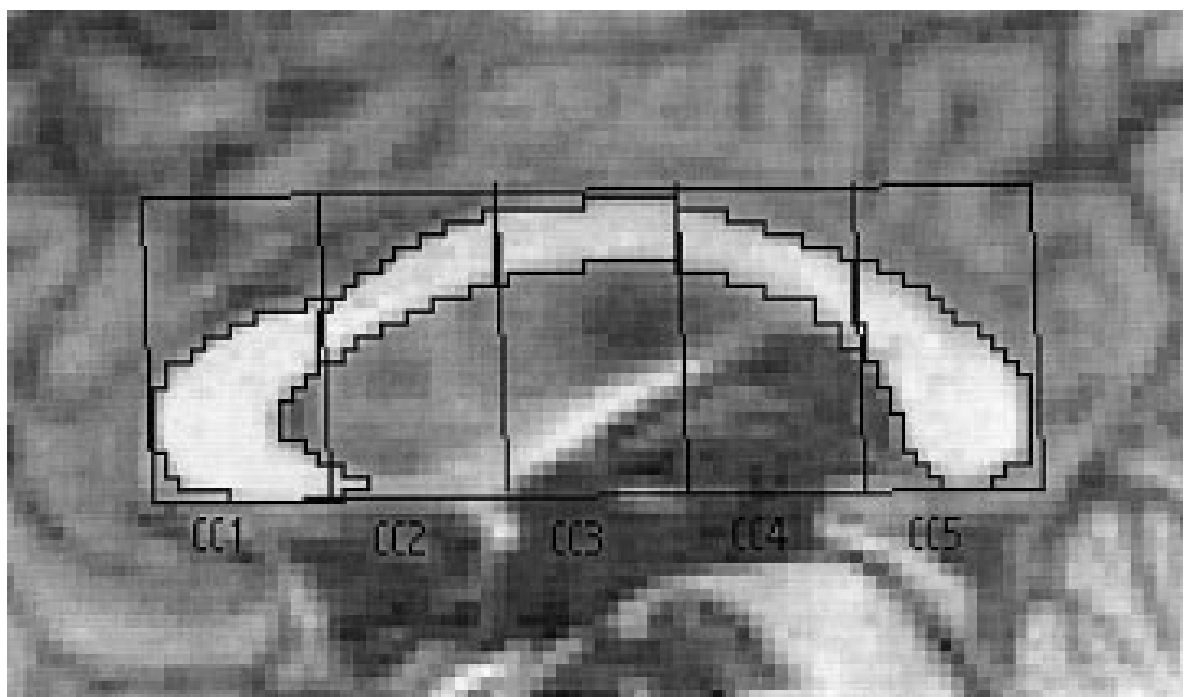

Figure 1 Manual outlining of callosal segments. A rectangle was constructed round the corpus callosum and divided into five parts of equal size (CC1 to CC5). CC1 incorporates the rostrum and genus, and CC5 the splenium.

dementia, and they were largest in the healthy subjects. This applies to raw and adjusted callosal areas. Repeated measures ANOVA for adjusted callosal areas showed a significant main effect of group $(\mathrm{F}[2,76]=4.594, \mathrm{p}=0.013)$. Post hoc LSD tests showed differences only between controls and mild dementia. In addition, segment 1 differed between questionable dementia and mild dementia. The raw and adjusted data are given in table 1 .

A significant main effect of sex was found. In all five callosal segments women had larger adjusted callosal areas than men $(\mathrm{F}[1,76]=4.438, \mathrm{p}=0.038)$. However, women and men did not differ in the relation between the three diagnostic groups for callosal size: group $\times \operatorname{sex}(F[2,76]=1.445, p=0.242)$.

No corpus callosum segment proved to be specially atrophied. Neither the diagnostic group nor sex nor age had a particular influence on a specific callosal segment: segment $\times$ group $(F[2,76]=1.494, p=0.231)$, segment $\times$ sex $(F[1,76]=0.685, p=0.411)$, segment $\times$ age $(F[1,76]=0.178$, $\mathrm{p}=0.281), \quad$ segment $\times$ group $\times \operatorname{sex} \quad(\mathrm{F}[2,76]=1.560$, $\mathrm{p}=0.217)$.
Discriminant analysis classified only $48.2 \%$ of the subjects correctly. Separate discriminant analyses for men and women did not improve the rate.

We found white matter changes in the majority of subjects. There was no group or sex difference by $\chi^{2}$ testing. Periventricular hyperintensities (pencil thin lining and smooth "halo") were present in 64 subjects and extended into the deep white matter in 12 subjects. Deep white matter hyperintensities occurred as punctuate foci and onset of confluence in 56 subjects, and as large confluent areas in eight subjects

\section{DISCUSSION}

Our study showed significant differences in callosal size between controls and subjects with mild dementia. This indicates that callosal atrophy is already present in mild Alzheimer's disease.

All five callosal subregions were found to be smallest in demented patients, followed by patients with questionable

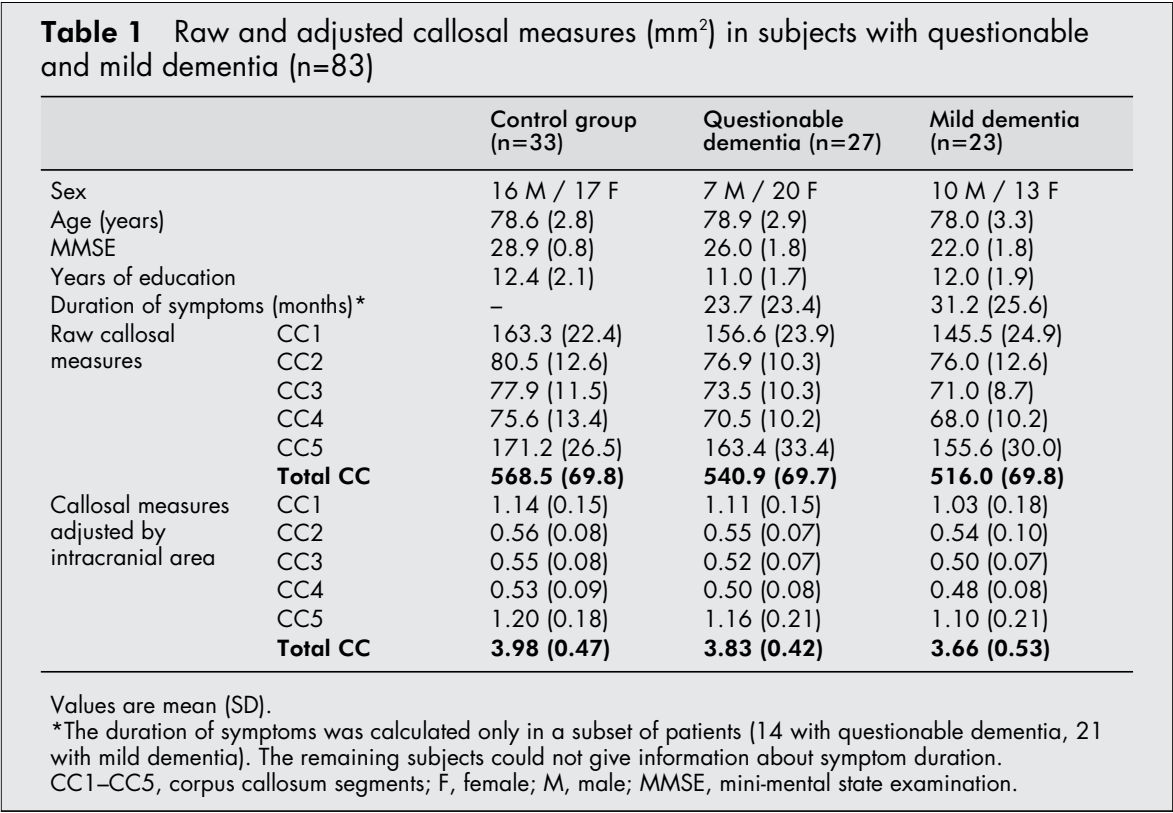


dementia, and largest in healthy subjects. However, no statistically significant differences were found between patients with questionable dementia and any other group. We may have missed truly significant differences owing to a lack of statistical power in our relatively small sample. Moreover, the group with questionable dementia might be too heterogeneous as to the cause and prognosis of their cognitive impairment. We do not know if the subjects identified as having "questionable dementia" will progress to true dementia or not, nor do we know if the underlying disease process is that of Alzheimer's disease. Previous studies have shown a high risk of progression to dementia of the Alzheimer type in subjects with a CDR of $0.5,{ }^{10}$ but there is also evidence of substantial heterogeneity in this difficult diagnostic subgroup. ${ }^{11}$

Our results are indirectly supported by data of Janowsky et $a l,{ }^{12}$ who studied elderly non-demented subjects with cognitive decline on two successive six month follow up visits. Corpus callosum size in these individuals was already apparently reduced at baseline, being intermediate between normal controls and demented patients, but it only differed significantly from the demented patients. Summarising the two studies, one might assume that a decline in the callosal area is a systematic feature of the transition from health to Alzheimer's disease.

We found the reduction in callosal size in our three diagnostic groups to be homogeneously distributed across the five segments. This was an unexpected finding. Previous studies comparing callosal atrophy in Alzheimer's disease patients and healthy subjects reported regional variation. ${ }^{13-15}$ However, the Alzheimer's disease groups investigated in those studies were more impaired and varied more in their cognitive performance than in ours. Regional specificity might occur only in the advanced stages of the disease.

Our results may have implications for the hypothesis that the pattern of callosal atrophy reflects the pattern of pathological changes in the cerebral neocortex. ${ }^{14}{ }^{16}$ According to the model of Braak and Braak, ${ }^{17}$ neocortical changes in mild Alzheimer's disease may occur in the temporal regions but may not be very marked. Such region specific changes were not reflected in region specific callosal atrophy in our study. The value of the corpus callosum as an index of neocortical changes may be limited in mild stages of Alzheimer's disease.

Our results support the relatively consistent finding that the adjusted corpus callosum area is larger in women than in men. However, our results produced no evidence for sex specific callosal atrophy in the early stages of Alzheimer's disease.

Our study shows that measurements of the corpus callosum are unsuitable for diagnostic differentiation between healthy individuals, those with questionable dementia, and those with mild Alzheimer's disease. Studies investigating the hippocampus have shown much higher rates of correct classification of up to $77 \% .{ }^{18}$ However, corpus callosum measurements may be useful in discriminating between different types of degenerative brain disease, as shown by Kaufer et al who achieved $85 \%$ correct classification in distinguishing between frontotemporal dementia and Alzheimer's disease. ${ }^{19}$

\section{Conclusions}

Our study showed a significant difference in the callosal area between normal controls and individuals with mild Alzheimer's disease. There was some indication that callosal atrophy could be a feature of the transition between health and Alzheimer's disease. However, callosal measurements were unsuitable for diagnostic differentiation between healthy subjects, subjects with questionable dementia, and subjects with mild Alzheimer's disease.

\section{ACKNOWLEDGEMENTS}

This study was supported by the Interdisziplinäres Zentrum für Klinische Forschung (IZKF) at the University of Leipzig (project C8).

\section{Authors' affiliations}

A Hensel, H Wolf, C Nikolaus, H J Gertz, Memory Clinic, Department of Psychiatry, University of Leipzig, Emilienstraße 14, 04107 Leipzig, Germany

F Kruggel, Max-Planck-Institute of Cognitive Neuroscience, Leipzig, Germany

S G Riedel-Heller, Research Department of Evaluation and

Epidemiology, University of Leipzig

T Arendt, Paul-Flechsig Institute for Brain Research, University of Leipzig

Correspondence to: DP A Hensel, Gedächtnissprechstunde, Universität Leipzig, Emilienstraße 14, 04107 Leipzig, Germany;

hensela@medizin.uni-leipzig.de

Received 25 July 2001

In revised form 11 March 2002

Accepted 28 March 2002

\section{REFERENCES}

1 Pantel J, Schroder J, Jauss M, et al. Topography of callosal atrophy reflects distribution of regional cerebral volume reduction in Alzheimer's disease. Psychiatry Res 1999;90:181-92.

2 Hughes CP, Berg L, Danziger WL, et al. A new clinical scale for the staging of dementia. BrJ Psychiatry 1982;140:566-72.

3 Roman GC, Tatemichi TK, Erkinjuntti T, et al. Vascular dementia: diagnostic criteria for research studies. Report of the NINDS-AIREN International Workshop. Neurology 1993;43:250-60.

4 Riedel-Heller SG, Schork A, Matschinger H, et al. Recruitment procedures and their impact on the prevalence of dementia. Results from the Leipzig Longitudinal Study of the Aged (LEILA75+). Neuroepidemiology 2000;19:130-40.

5 Zaudig M, Mittelhammer J, Hiller W, et al. SIDAM - a structured interview for the diagnosis of dementia of the Alzheimer type, multi-infarct dementia and dementias of other aetiology according to ICD-10 and DSM-III-R. Psychol Med 1991;21:225-36.

6 Folstein MF, Folstein SE, McHugh PR. "Mini-mental state". A practical method for grading the cognitive state of patients for the clinician. $J$ Psychiatr Res 1975;12:189-98.

7 Kruggel F, Lohmann G. BRIAN - a toolkit for the analysis of multimodal brain datasets. In: Lemke HU, Inamura K, Jaffe CC, et al, eds. Computer assisted radiology. Paris: Springer Heidelberg, 1996:323-8.

8 Talairach J, Tournoux, P. Co-planar stereotactic atlas of the human brain. Stuttgart: Thieme, 1988

9 Weis S, Jellinger K, Wenger E. Morphometry of the corpus callosum in normal aging and Alzheimer's disease. J Neural Transm Suppl 1991;33:35-8

10 Rubin EH, Morris JC, Grant EA, et al. Very mild senile dementia of the Alzheimer type. I. Clinical assessment. Arch Neurol 1989;46:379-82.

11 Wolf $\mathbf{H}$, Grunwald M, Ecke GM, et al. The prognosis of mild cognitive impairment in the elderly. J Neural Transm Suppl 1998;54:31-50.

12 Janowsky JS, Kaye JA, Carper RA. Atrophy of the corpus callosum in Alzheimer's disease versus healthy aging. J Am Geriatr Soc 1996;44:798-803.

13 Biegon A, Eberling JL, Richardson BC, et al. Human corpus callosum in aging and Alzheimer's disease: a magnetic resonance imaging study. Neurobiol Aging 1994;15:393-7.

14 Hampel H, Teipel SJ, Alexander GE, et al. Corpus callosum atrophy is a possible indicator of region- and cell type-specific neuronal degeneration in Alzheimer disease: a magnetic resonance imaging analysis. Arch Neurol 1998;55:193-8.

15 Teipel SJ, Hampel H, Alexander GE, et al. Dissociation between corpus callosum atrophy and white matter pathology in Alzheimer's disease. Neurology 1998:51:1381-5.

16 Yamauchi H, Fukuyama H, Nagahama Y, et al. Comparison of the pattern of atrophy of the corpus callosum in frontotemporal dementia, progressive supranuclear palsy, and Alzheimer's disease. J Neurol Neurosurg Psychiatry 2000;69:623-9.

17 Braak H, Braak E. The human entorhinal cortex: normal morphology and lamina-specific pathology in various diseases. Neurosci Res 1992;15:6-31.

18 Wolf $\mathbf{H}$, Grunwald M, Kruggel F, et al. Hippocampal volume discriminates between normal cognition; questionable and mild dementia in the elderly. Neurobiol Aging 2001;22:177-86.

19 Kaufer DI, Miller BL, Itti L, et al. Midline cerebral morphometry distinguishes frontotemporal dementia and Alzheimer's disease. Neurology 1997;48:978-85. 